\title{
Le chant du départ de Marie-Joseph Chénier et Etienne Méhul
}

Jean-François Domine

\section{(2) OpenEdition \\ 12 Journals}

Édition électronique

URL : https://journals.openedition.org/ahrf/701

DOI : 10.4000/ahrf.701

ISSN : 1952-403X

Éditeur :

Armand Colin, Société des études robespierristes

Édition imprimée

Date de publication : 1 septembre 2002

Pagination : 89-100

ISSN : 0003-4436

\section{Référence électronique}

Jean-François Domine, «Le chant du départ de Marie-Joseph Chénier et Etienne Méhul », Annales

historiques de la Révolution française [En ligne], 329 | juillet-septembre 2002, mis en ligne le 27 mars 2008, consulté le 23 avril 2022. URL : http://journals.openedition.org/ahrf/701 ; DOI : https://doi.org/ 10.4000/ahrf.701 


\title{
LE CHANT DUDÉPART DE MARIE-JOSEPH CHÉNIER ET ETIENNE MÉHUL
}

\author{
JEAN-FRANÇOIS DOMINÉ
}

\begin{abstract}
CEuvre de circonstance, Le Chant du départ a été joué dans plusieurs cérémonies officielles avant d'être mis à l'écart par Napoléon 1er. La mise en scène (des personnages s'interpellent) comme la structure en strophes et la versification lui confèrent une allure solennelle que renforce l'idéologie volontariste et manichéenne du texte. La comparaison souvent conduite avec $\mathrm{La}$ Marseillaise aboutit au constat que la prééminence de l'une sur l'autre provient autant des deux hymnes eux-mêmes que de l'histoire (l'antériorité de La Marseillaise, son rôle social jusqu'à la venue de I'Internationale en 1888) alors qu'ils ont connu des destins parallèles.
\end{abstract}

Mots clés : hymne révolutionnaire ; Le Chant du départ; La Marseillaise ; Marie-Joseph Chénier ; Rouget de l'Isle.

Parmi les productions de caractère politique (tract, affiche, slogan, article, discours) les hymnes et les chansons occupent une place à part. Mélange d'affectivité et d'idéologie, ils relèvent davantage de la première et surgissent en général dans des circonstances particulièrement dramatiques (excepté les cérémonies officielles bien entendu). Cette spécificité confère tout son intérêt à leur étude.

Le présent article comprend trois parties. Il propose en premier lieu une analyse du Chant $d u$ départ aussi large que possible puisqu'elle aborde successivement : la composition (A) la versification (B) la grammaire syntaxe, morphologie, ponctuation - (C) le lexique (D) ainsi que les figures de rhétorique (E) ; suivent quelques jugements sur Le Chant du départ. Est esquissée enfin une comparaison entre La Marseillaise et Le Chant du départ dont les textes figurent en annexe.

Mais rappelons tout d'abord comment est né Le Chant du départ. Bien qu'il s'agisse d'une œuvre illustre, sa naissance est en effet assez mal connue. Selon le Larousse Universel, il a été composé à la demande de 
Sarrette, directeur de l'Institut national de musique afin de commémorer le cinquième anniversaire de la prise de la Bastille. D'autres raisons ont toutefois été avancées. Marie-Joseph Chénier aurait écrit ce texte, mis en musique par Méhul vers juin 1794, pour faire sortir son frère André de prison ou échapper lui-même aux menaces de la loi du 22 prairial sur les suspects. Au demeurant, Sarrette étant pour sa part suspect d'hébertisme, son initiative pouvait lui valoir l'indulgence de la Convention. Enfin, l'hymne a pu être conçu pour célébrer les victoires de l'an II et notamment celle, toute récente, de Fleurus (26 juin 1794).

Il est certain, en revanche, que Le Chant du départ fut créé «lors de la fête destinée à célébrer la prise d'Ostende qui se déroula à Paris, au Jardin national (Jardin des Tuileries) le 16 messidor an II (4 juillet 1794)».

Par la suite, il fut exécuté au cours de plusieurs cérémonies officielles : le transfert des cendres de Marat au Panthéon (21 septembre 1794); la Fête des Victoires et de l'École de Mars (31 octobre 1794); l'anniversaire de la mort du roi (21 janvier 1795).

Selon René Brancour (La Marseillaise et Le Chant du départ, Henri Laurens, collection Images historiques, Paris, 1915) «Napoléon aimait beaucoup Le Chant $d u$ départ qu'il fredonnait volontiers. Il [Le Chant $d u$ départ] accompagna la première distribution des croix de la Légion d'honneur au camp de Boulogne en $1804 »$.

\section{ANALYSE DU TEXTE}

\section{A. La composition}

Le Chant du départ est formé de sept strophes de huit vers chacune séparées par un refrain de quatre vers. Chaque strophe met en scène un personnage qui symbolise un élément de la société républicaine telle qu'elle est décrite dans Les fragments d'institutions républicaines de Saint-Just : représentant du peuple, guerriers, mère de famille, vieillards, enfant, épouse, sœur. Les femmes et les vieillards incitent les hommes à faire preuve de courage patriotique.

\section{B. La versification}

Très classiquement, le premier et le troisième vers de chaque strophe sont des alexandrins, le second et le quatrième des octosyllabes. Les rimes sont croisées (abab); le refrain a ses rimes propres, chaque strophe en 
contient deux séries (cdcd/efef). L'alternance masculin/féminin est en général respectée, notamment dans le refrain mais les pluriels abondants ne le permettent pas toujours. Il s'agit de rimes suffisantes mais souvent banales (larmes/armes, guerriers/lauriers, pères/mères). Sont à noter les nombreux monosyllabes en fin de vers (pas; vie; vous; nous) ainsi que le $s$ muet de Mars dans la troisième strophe. Les sonorités claires alternent avec des sons plus sourds; certaines sont employées à deux reprises (-u, -ière) et même à trois (-eurs).

\section{La grammaire}

\section{a. La syntaxe}

Chaque strophe est formée de deux phrases ou de deux propositions indépendantes reliées par «et». On relève également deux temporelles et deux conditionnelles. Seule, la $6^{\mathrm{e}}$ strophe présente une anacoluthe (une rupture dans la construction syntaxique) plus inattendue. En effet, il manque au membre de phrase initial «Et nous, sœurs des héros, nous, qui de l'hyménée/Ignorons les aimables nœuds, » le verbe censé lui donner un sens.

\section{b. La morphologie}

\section{Les verbes}

L'ensemble du texte est à l'indicatif, le plus souvent présent (dix occurrences) mais aussi passé composé (cinq) et futur (trois). La $5^{\mathrm{e}}$ strophe contient une concordance peu habituelle : si et l'imparfait suivis du futur. L'emploi du verbe «être » (huit) du gérondif (deux) et des infinitifs compléments traduit le souci de l'auteur de donner une portée générale à son propos. On dénombre enfin quinze impératifs, dont la signification est étudiée au paragraphe $\mathrm{E}$, les figures de rhétorique, ci-dessous.

\section{Les substantifs}

L'emploi quasi systématique du pluriel montre lui aussi la volonté de généralisation évoquée ci-dessus.

\section{c. La ponctuation}

Les onze points correspondent le plus souvent aux fins des strophes et $\mathrm{du}$ refrain mais le texte est parcouru de virgules (trente-cinq) et de pointsvirgules (vingt); s'y ajoutent deux deux-points explicatifs et deux points d'exclamation associés à des impératifs. 


\section{Le lexique}

Il se caractérise par la fréquence des noms abstraits (liberté, égalité, destinée...) et par le recours au pluriel mentionné plus haut. Les mots concrets déclinent le thème du combat héroïque dont l'alternative est la victoire (fleurs, lauriers) ou la mort (blessures, mânes). Il n'y a que deux noms propres, Bara et Viala, jeunes héros dont la Convention a voté l'entrée au Panthéon; la romance patriotique sur la mort du jeune Bara (paroles de Dossion) développe l'idée brièvement évoquée au début de la 4 e strophe : mourir pour la patrie est un destin enviable. C'est donc un lieu commun de l'époque que reprend ici Chénier.

Les épithètes sont plus souvent postposés (sept) qu'antéposés (trois). On remarque l'emploi de «vainqueurs » pour « victorieux » sans doute pour des raisons techniques (nombre de pieds, rime) ainsi que l'association adjectif/de/nom abstrait («beaux de gloire et de liberté ») alors qu'on rencontre plus souvent la formule adjectif/de/nom concret (vert de peur, rouge de colère, fou de joie).

Nombreux sont les adjectifs possessifs (douze «nos» ou «notre », huit «vos» ou «votre »). Le pronom «nous» revient quatorze fois, mais «vous» quatre fois seulement et «ils» trois. Cette fréquence de la 1re personne du pluriel met en valeur la cohésion du groupe formé par les personnages et la solidarité entre ses membres.

Peu d'adverbes ou de locutions adverbiales : « en tous lieux », « loin ».

Les mots-outils les plus fréquents sont «à », « de », « et ».

\section{E. Les figures de rhétorique}

On peut distinguer schématiquement quatre groupes de figures de rhétorique : les figures de pensée modifient la valeur logique de la phrase mais ne dépendent pas de processus sémantiques particuliers; les figures de style agissent sur la structure de la phrase; les tropes opèrent des changements d'ordre sémantique dans le mot; les figures de diction jouent sur l'aspect graphique ou sonore du mot.

Dans Le Chant du départ, les premières nommées sont prédominantes.

Parmi elles, l'optation qui exprime un vœu sous une forme incantatoire, apparaît quinze fois. Or, cette figure se manifeste de plusieurs manières : l'impératif (dix), l'expression «il faut que » abrégée en «que » (trois), le verbe «devoir» (deux); on peut y inclure le verbe «jurer» (7e strophe) qui constitue un impératif moral. Ce recours à l'optation met en évidence le volontarisme développé par l'hymne. 
Est très présente également l'apostrophe (six fois). Cela est dû à la composition même du Chant du départ où les personnages s'interpellent et se répondent.

À la frontière entre les figures de pensée et les figures de style, l'antithèse consiste à opposer deux termes (mots, idées, personnes). C'est une figure très employée dans le discours politique en particulier sous la Révolution; elle peut être résumée par : «nous et eux » ou bien « les bons et les méchants». Ici, elle apparaît notamment dans le refrain («Sachons vaincre ou sachons périr ») dont les deux derniers vers forment un chiasme (figure combinant l'antithèse et l'inversion); mais les conjonctions ou adverbes tels que «mais » et «cependant», ou encore la symétrie en sont d'autres variantes («Ils [Bara, Viala] sont morts mais ils ont vaincu »; «Le lâche accablé d'ans n'a point connu la vie/Qui meurt pour le peuple a vécu »; «Les républicains sont des hommes/Les esclaves sont des enfants ».

Les seules tropes sont des métaphores usées (dites aussi mortes) c'est-à-dire des images si souvent utilisées qu'elles ne sont plus ressenties comme telles : «les combats sont vos fêtes »; le fer pour l'épée (deux fois); les «champs de Mars» pour les champs de bataille, «le temple de mémoire » pour le cimetière ; «nos flancs portent vos vengeurs » désigne les futurs enfants des guerriers.

L'usage de la prosopopée (qui consiste à faire parler des êtres inanimés) en l'occurrence la Victoire, la République, la Liberté, confère au Chant du départ une sorte de théâtralité, de solennité culminant dans la dernière strophe.

\section{QUELQUES JUGEMENTS SUR LE CHANT DU DÉPART}

Dans l'Histoire des Girondins (chapitre XIII, livre 56 e , pp. 774/775) Lamartine écrit : «Ainsi la République triomphait ou s'illustrait partout. La Convention appelait tous les arts et tous les génies à célébrer ces premiers triomphes de la liberté. Comme les périls de 1793 avaient leur Tyrtée (1) dans Rouget de l'Isle, les victoires de 1794 avaient le leur dans M.-J. Chénier et dans Lebrun. Ce fut alors que Chénier composa Le Chant du départ dont les mots respiraient le triomphe comme ceux de La Marseillaise respiraient la fureur. »

(1) Poète lyrique grec (Attique, VIIe siècle av. J.-C.) qui ranima par ses chants le courage des Spartiates pendant la seconde guerre de Messénie. 
Julien Tiersot dans Les fêtes et les chants de la Révolution française (Paris, Librairie Hachette et $\mathrm{Ci}^{\mathrm{e}}$, 1908) chapitre VII, «L'art républicain en l'an II : Le Chant du départ», note que si l'on a pu appeler Le Chant du départ «la seconde Marseillaise », «ce n'est point là un titre usurpé : on retrouve dans cette œuvre splendide presque l'équivalent de l'inspiration de la flamme qui a créé le chant national. Elle en a presque la spontanéité ; elle traduit aussi fidèlement l'aspiration du temps qui l'a vu naître. La Marseillaise c'était déjà un Chant du départ, le départ pour la guerre à son premier jour : maintenant deux ans ont passé et Chénier date son nouveau poème quand il commence par ce vers admirable : «La Victoire, en chantant, nous ouvre la barrière ». Toutefois, Tiersot observe que «si beau que soit le Chant du départ, il n'égale pas La Marseillaise. Par l'ardeur, le jaillissement de l'inspiration, le chant du soldat est bien supérieur à celui de l'artiste. Tous deux possèdent les mêmes qualités mais, chez Méhul, la spontanéité est tempérée par les habitudes d'école : rien, au contraire, n'en entrave la manifestation chez Rouget de l'Isle. »

René Brancour (op. cit.) oppose « l'irrésistible élan... la couleur romantique » [de La Marseillaise] à « la sereine noblesse... la pureté de lignes classique » [du Chant du départ].

G. et G. Marty considèrent Le Chant du départ comme « un hymne de guerre où, tour à tour, les mères, les vieillards, les épouses excitent le zèle et le courage des citoyens et font appel à leur dévouement pour la défense de la patrie » (Dictionnaire des chansons de la Révolution, 1988, p. 187).

En 1989, Frédéric Robert a consacré un dossier remarquablement illustré et documenté à La Marseillaise (édité par l'Imprimerie nationale); y figurent plusieurs analyses de l'hymne envisagé sous divers angles. Ainsi, Eugène Géruzez (2) le compare au Chant du départ. «La Révolution eut donc à combattre ceux-là même qu'elle voulait délivrer, et comme si l'hymne de Rouget de l'Isle n'eût pas suffi à enflammer les courages, Chénier y ajouta Le Chant du départ, mieux composé, mais bien plus pâle à côté de La Marseillaise, et inutile pour la lutte. C'est une scène lyrique animée de nobles sentiments où l'art se montre plus que la verve et qui n'a pas dû inspirer beaucoup de crainte aux tyrans, quoique le poète se fasse terrible et qu'il enfle sa voix pour les menacer. En vain leur crie-t-il :

"Tremblez, ennemis de la France,

Rois ivres de sang et d'orgueil !

Le peuple souverain s'avance :

Tyrans, descendez au cercueil."

(2) Passage extrait de l'Histoire de la littérature française pendant la Révolution, 1789-1800, 6 édition, G. CHARPENTIER, 1877, pp. 191-194. 
Ce peuple souverain s'avance trop majestueusement pour les y pousser et ils n'y descendent pas d'eux-mêmes. Que vaut cette pompeuse poésie auprès du cri martial : "Aux armes, citoyens ! Formez vos bataillons !" Cela parle au cœur du soldat et retentit comme les clairons.

Chénier fait chanter un représentant du peuple, une mère de famille, deux viellards, un enfant, une épouse, une jeune fille, trois guerriers et n'entraînera personne ; quand l'épouse chante :

"Partez, vaillants époux, les combats sont vos fêtes ;

Nous cueillerons des fleurs pour en ceindre vos têtes ;

Nos mains tisseront vos lauriers ;"

Les époux si mélodieusement congédiés devaient être moins pressés de partir. Rouget de l'Isle n'avait poussé qu'un cri et tout un peuple s'était levé. »

Le Dictionnaire des auvres de l'art vocal (tome A-F, Dir. Marc Honegger et Paul Prévost, Bordas, 1991) estime que Le Chant du départ est « une des plus célèbres chansons patriotiques de la Révolution... composée pour commémorer les victoires françaises de l'an II. Pendant un certain temps, elle rivalisa en popularité avec La Marseillaise. Si le texte fait allusion à des événements récents comme le martyre des jeunes enfants Bara et Viala, les sentiments exprimés ont en général une portée plus universelle : l'amour de la patrie et la nécessité de défendre la liberté et la République. Méhul les a traités avec intelligence : la mélodie, facile à retenir, a un caractère vigoureux et, quand les paroles l'exigent, elle a souligné le contraste entre les rois ennemis de la République et les braves citoyens par un changement de mode. Le refrain est un remarquable appel aux armes. »

\section{ffl. LA MARSEILLAISE ET LE CHANT DU DÉPART}

Il est tentant de comparer La Marseillaise et Le Chant du départ et cinq des six commentaires qui précèdent se livrent à cet exercice. De fait, les deux hymnes ont plus d'un point commun : ils sont contemporains et ont bénéficié d'une durable notoriété. En outre, tous deux sont d'une inspiration très proche dans la forme (une structure presque identique, une versification classique) comme dans le fond (un discours polémique et volontariste qui s'exprime à travers les figures marquantes de l'optation et de l'antithèse, un vocabulaire tiré du fonds commun de l'époque). Néanmoins, $L a$ Marseillaise a conservé une prééminence incontestée sur Le Chant du départ. Voyons pourquoi.

Les études précitées fournissent une première clé. Leurs auteurs, sauf peut-être Lamartine, attribuent à La Marseillaise un souffle épique qui manque au Chant du départ en dépit des indéniables qualités esthétiques de 
ce dernier. Ainsi, l'invitation du refrain est-elle directe : «Aux armes, citoyens, formez vos bataillons », concrète : «Marchons, marchons » et victorieuse : «Qu'un sang impur abreuve nos sillons » et non indirecte : « La République nous appelle », abstraite : «Sachons vaincre ou sachons périr », et ambiguë : «Un Français doit vivre pour elle/Pour elle un Français doit mourir. »

En outre, La Marseillaise est plus homogène. Le Chant du départ présente un groupe de personnages d'âge et de sexe différents qui s'interpellent. Cette mise en scène ne permet pas à une foule de manifestants d'entonner l'hymne en chœur contrairement à La Marseillaise. Les textes contiennent donc ce handicap, encore que le mot soit un peu fort, en germe.

La chronologie milite également en faveur de La Marseillaise : créée deux ans environ avant Le Chant du départ elle occupe un espace quasiment vide (à l'exception du Ça ira et de La Carmagnole) ; ensuite, elle a été adoptée d'emblée par les volontaires marseillais qui la font connaître partout où ils passent. De la même manière, les guerres napoléoniennes la rendront célèbre dans toute l'Europe.

Ce n'est pas tout. La Marseillaise, du moins jusqu'à ce que L'internationale tienne ce rôle à partir de 1888, est un chant de caractère social. Dans un travail récent, «La Marseillaise, étude sociologique de l'hymne national ; Glossaire historique » (ministère de la Défense, secrétariat d'État aux anciens combattants, co-production avec les Éditions Crimée, 1997) M. Michel Prévot distingue «La Marseillaise héroïque», «La Marseillaise républicaine et sociale » et «La Marseillaise de la Nation » qui, chacune à sa manière, expriment la pérennité des valeurs de la Nation.

Cette ductilité (elle peut être revendiquée comme chant patriotique, révolutionnaire ou social) de La Marseillaise ajoutée aux raisons techniques indiquées plus haut explique pourquoi Le Chant du départ a été devancé par La Marseillaise. Pourtant, leurs destins sont parallèles. Schématiquement, ils ont connu des traversées du désert sous les régimes autoritaires et des retours en grâce au cours de périodes révolutionnaires ; mais la force symbolique de La Marseillaise lui a valu les plus durs revers mais aussi les plus glorieux réveils. Ce fut le cas sous l'Occupation et à la Libération.

Deux exemples montrent que Le Chant du départ n'a jamais obtenu une véritable reconnaissance populaire. On se souvient peut-être que M. Giscard d'Estaing a, au début de son septennat (1974-1981) proposé de remplacer la première par le second comme hymne national sans aucun succès. Sur un autre registre, il n'existe qu'une trentaine d'imitations du Chant du départ \{Dictionnaire historique de la Révolution française, ss dir. Albert Soboul, PUF, 1989) alors que La Marseillaise a suscité une centaine d'adaptations (mentionnées par M. Prévot, op. cit., p. 77). 
La Marseillaise a supplanté le Ça ira et La Carmagnole ainsi que l'impérial Veillons au salut de l'Empire. Sa seule vraie rivale a été L'Internationale, près d'un siècle sa cadette. Hymne national depuis le 14 février 1879 sans interruption hormis l'épisode vichyste, La Marseillaise a toujours été plus populaire que Le Chant du départ. Même si les deux hymnes sont des œuvres de circonstance, La Marseillaise a été conçue dans l'enthousiasme par un militaire amateur de musique et de poésie alors que Le Chant $d u$ départ a été élaboré par deux artistes de profession Ce n'est pas faire injure à M.-J. Chénier et Méhul que d'interpréter ce succès comme la victoire de la sincérité sur le calcul, de la simplicité sur l'artifice et du génie sur le talent.

Jean-François DOMINÉ

137, boulevard Auguste Blanqui

75013 Paris

\section{ANNEXE}

Textes des deux hymnes tels qu'ils sont reproduits dans le Chansonnier révolutionnaire (NRF, Poésie/Gallimard, 1989) respectivement $n^{\text {os }} 97$ et 32. L'ouvrage comporte un excellent appareil critique.

\section{LE CHANT DU DÉPART}

Un représentant du peuple

La Victoire, en chantant, nous ouvre la barrière ;

La liberté guide nos pas ;

Et, du nord au midi, la trompette guerrière

A sonné l'heure des combats.

Tremblez, ennemis de la France,

Rois ivres de sang et d'orgueil ;

Le peuple souverain s'avance :

Tyrans, descendez au cercueil.

La République nous appelle ;

Sachons vaincre ou sachons périr ;

Un Français doit vivre pour elle ;

Pour elle un Français doit mourir.

Le chœur des guerriers

La République nous appelle, etc. ; 


\section{Une mère de famille}

De nos yeux maternels ne craignez point les larmes ;

Loin de nous les lâches douleurs !

Nous devons triompher quand vous prenez les armes ;

C'est aux rois à verser des pleurs.

Nous vous avons donné la vie;

Guerriers, elle n'est plus à vous :

Tous vos jours sont à la patrie ;

Elle est votre mère avant nous.

\section{Chœur des mères de famille}

La République vous appelle, etc.

\section{Deux vieillards}

Que le fer paternel arme la main des braves;

Songez à nous aux champs de Mars :

Consacrez dans le sang des rois et des esclaves

Le fer béni par vos vieillards ;

Et, rapportant sous la chaumière,

Des blessures et des vertus,

Venez fermer notre paupière

Quand les tyrans ne seront plus.

\section{Chœur des vieillards}

La république nous appelle, etc.

\section{Un enfant}

De Bara, de Viala, le sort nous fait envie ;

Ils sont morts mais ils ont vaincu :

Le lâche accablé d'ans n'a point connu la vie ;

Qui meurt pour le peuple a vécu.

Vous êtes vaillants, nous le sommes ;

Guidez-nous contre les tyrans :

Les républicains sont des hommes ;

Les esclaves sont des enfants.

\section{Chour des enfants}

La République nous appelle, etc.

\section{Une épouse}

Partez, vaillants époux, les combats sont vos fêtes ;

Partez, modèles des guerriers ;

Nous cueillerons des fleurs pour en ceindre vos têtes ;

Nos mains tresseront vos lauriers ;

Et si le temple de mémoire

S'ouvrait à vos mânes vainqueurs,

Nos voix chanteront votre gloire

Et nos flancs portent vos vengeurs. 


\section{Chœur des épouses}

La République vous appelle, etc.

\section{Une jeune fille}

Et nous, sœurs des héros, nous qui de l'hyménée

Ignorons les aimables nœuds.

Si pour s'unir un jour à notre destinée

Les citoyens forment des vœux,

Qu'ils reviennent dans nos murailles

Beaux de gloire et de liberté,

Et que leur sang dans les batailles

Ait coulé pour l'égalité !

\section{Chœur des jeunes filles}

La République vous appelle, etc.

\section{Trois guerriers}

Sur le fer, devant dieu, nous jurons à nos pères,

À nos épouses, à nos sœurs,

À nos représentants, à nos fils, à nos mères,

D'anéantir les oppresseurs.

En tous lieux, dans la nuit profonde

Plongeant la féodalité,

Les Français donneront au monde

Et la paix et la liberté.

\section{Chœur général}

La République nous appelle, etc.

\section{LA MARSEILLAISE}

Allons, enfants de la patrie,

Le jour de gloire est arrivé

Contre nous de la tyrannie

L'Étendard sanglant est levé (bis)

Entendez-vous dans les campagnes

Mugir ces féroces soldats

Ils viennent jusque dans vos bras

Égorger vos fils, vos compagnes.

Aux armes, citoyens !

Formez vos bataillons !

Marchez, marchez!

Qu'un sang impur

Abreuve nos sillons. 
Que veut cette horde d'esclaves,

De traîtres, de rois conjurés?

Pour qui ces ignobles entraves,

Ces fers dès longtemps préparés ? (bis)

Français, pour nous, ah! quel outrage,

Quels transports il doit exciter?

C'est nous qu'ose méditer

De rendre à l'antique esclavage ! (refrain)

Quoi, des cohortes étrangères

Feraient la loi dans nos foyers ?

Quoi, ces phalanges mercenaires

Terrasseraient nos fiers guerriers ? (bis)

Grand Dieu !... Par des mains enchaînées,

Nos fronts sous le joug ploieraient,

Des vils despotes deviendraient

Les maitres de nos destinées ? (refrain)

Tremblez, tyrans ! et vous, perfides

L'opprobre de tous les partis,

Tremblez... vos projets parricides

Vont enfin recevoir leur prix. (bis)

Tout est soldat pour vous combattre

S'ils tombent, nos jeunes héros,

La terre en produit des nouveaux

Contre vous tous prêts à se battre.(refrain)

Français, en guerriers magnanimes,

Portez ou retenez vos coups,

Épargnez ces tristes victimes

À regret s'armant contre nous. (bis)

Mais le despote sanguinaire,

Mais les complices de Bouillé,

Tous ces tigres qui, sans pitié,

Déchirent le sein de leur mère. (refrain)

Amour sacré de la patrie

Conduis, soutiens nos bras vengeur

Liberté, liberté chérie,

Combats avec tes défenseurs. (bis)

Sous nos drapeaux, que la victoire

Accoure à tes mâles accents

Que tes ennemis expirants

Voient ton triomphe et notre gloire! (refrain) 